\title{
LAS DOS EDICIONES DEL AMINTA DE JÁUREGUI
}

Don Juan de Jáuregui emprendió su célebre traducción del Aminta de Tasso durante su permanencia en Roma, y la publicó en esta misma ciudad, en casa de Estevan Paulino, el año de 1607. Es indudable que la edición del original italiano que tuvo a la vista fue la aldina de 1590 , ya que su versión reproduce tres peculiaridades que aparecen en esa edición de 1590 y que no se encuentran en ninguna otra, a saber: I) la palabra protesto en el verso 988 (las demás impresiones italianas dicen allí pretesto); 2) la lección "come colui ch'impara" en el verso $115^{\circ}$ ("come colà s'impara" en las demás ediciones); 3) los versos 1470-78, "Non bisogna la morte..." (tercer coro), en cuyo lugar aparece, en el resto de las ediciones antiguas del Aminta italiano, un madrigal distinto, "Gelo ha madonna in seno..."; Jáuregui reproduce en su versión el protesto del v. 988, traduce normalmente el v. 1150 , "al fin como el qu'enseñan", y traduce asimismo el tercer coro en la forma que presenta la edición aldina de 1590 .

Once años después, en 1618 , se imprimió en Sevilla una segunda edición del Aminta castellano', en la cual introdujo el traductor, como luego veremos, buen número de enmiendas. Mantuvo, sin embargo, la lección protesto; conservó también el tercer coro, y en cuanto al v. 1150, se limitó a retocarlo en "parece que le enseñan", pero sin tener en cuenta el colà de la generalidad de las ediciones italianas. Ahora bien, esta impresión sevillana nos plantea un pequeño problema: a diferencia de la edición romana de 1607 , no aparece en ella el episodio de Mopso, al final del primer acto. Cuando Sedano reprodujo el Aminta de Jáuregui en el primer tomo de su Parnaso español (Madrid, 1768), lamentó justamente esa omisión, y supuso que tal vez a Jáuregui "le pareció algo impertinente [el episodio de Mopso], o más propio de un poema épico que del dramático" (op. cit., pp. xxi-xxii). Yo creo, sin embargo, que la explicación es otra. Muy probablemente el traductor sabía que Tasso

1 Cito directamente la primera versión por la edición romana de 1607 ; para la segunda versión me sirvo del texto de Sedano, en el primer tomo del Parnaso español. Hago las citas italianas de acuerdo con la edición del Aminta por $\mathbf{B}$. T. Sozzi, Padova, 1957. 
mismo había tenido ciertas vacilaciones en cuanto a la conservación o supresión de ese episodio, el cual no figura en dos de las ediciones hechas en vida del poeta, una de Cremona (Draconi, $15^{80}$ ) y otra de Venecia (Aldo, 1581). En otras palabras, Jáuregui pudo servirse de una de estas dos ediciones, fundándose en ella para la supresión del episodio de Mopso, pero sin utilizarla para retocar los tres pasajes arriba mencionados.

En su carta dedicatoria a don Fernando Enríquez de Ribera, duque de Alcalá, Jáuregui llama "traslación" a la versión poética por él realizada. Este término, un tanto impreciso, queda aclarado en cierto modo cuando más adelante se refiere Jáuregui al sentido de la palabra "transferir": su propósito ha sido proporcionarnos una imagen de su modelo por medio de una traducción literal, dentro de las limitaciones expresivas que le imponía la elegancia estilística y el buen uso castellano. La tarea se le facilitó por la íntima afinidad de léxico que existe entre las dos lenguas, afinidad que a veces hace posible el trasiego casi perfecto de versos cuyas palabras tienen la misma raíz en las dos lenguas, como se ve en los ejemplos siguientes:

selvaggio o de la plebe de gli dei e solo al vulgo de' ministri miei se io che son l'Amor d'amor m'intendo ma che non puote il tempo? e che non [puote servendo, meritando, supplicando...? d'un secreto veleno

e le virtù de l'erbe e de le fonti quivi abitan le maghe che incantando ma la replican tutta intiera intiera non moverei per aiutarti un passo negligente maestra, perchè solo selvaje o de la plebe de los dioses i sólo al vulgo de ministros míos si yo que soy Amor d'amor entiendo mas ¿qué no puede el tiempo? y ¿qué [no puede sirviendo, mereciendo y suplicando...? de un secreto veneno

la virtud de las yerbas y las fuentes aquí habitan las magas que encantando mas la replican toda entera entera no moveré por ayudarte un paso negligente maestra, ¿por qué sólo

Hay por lo menos cien versos de este tipo.

Donde no fue posible llevar a cabo una reproducción perfecta o casi perfecta de todo un endecasílabo, Jáuregui procuró conservar integramente una de las unidades rítmicas de su original:

non riconoscerà sì di leggiero che questo è il nome de l'alpestre ninfa come a me piace agguagli: e questa è [pure atterrar combattendo; e, se non man. [cano

la sanguinosa spada: ed a Nettuno scotitor de la terra il gran tridente che mai seguisse il coro di Diana suprema gloria e gran miracol mio Mostrommi l'ombra d'una breve notte di mille giorni non m'avea mostrato no reconocerá tan fácilmente que éste es el nombre de la ninfa fiera como me place igualo; ésta es la suma

aterrar combatiendo la más brava

de la robusta mano; y a Neptuno que las tierras combate, el gran tridente siguió jamás el coro de Diana gloria que alcanzo: el gran milagro mio la oscura sombra de una breve noche en largo tiempo no me habia mostrado 
A veces la unidad rítmica que aparece en la parte inicial del verso italiano queda colocada en la última porción del de Jáuregui:

la sanguinosa spada; ed a Nettuno le rustiche sampogne; e, se mia madre in ogni breve spazio? or sotto all'ombre

Amor servo de l'oro è il maggior mostro derriba a Marte la sangrienta espada que suelo hacer las rústicas zampoñas Como el Amor que en todo breve [espacio

Amor vendible, amor siervo del oro

Hay otros casos en que el procedimiento es inverso:

ciò non conosce, e cieca ella $\dot{e}$, non io albergo gli dineghi ne'l tuo petto Ben riconosco in voi gl'inganni vostri sì ch'insieme movea pietate e riso ella es ciega, no yo, que falsamente le niegas en tu pecho acogimiento y los engaños vuestros; mas qué importa piedad y risa en ninfas y pastores

He escogido deliberadamente los ejemplos homólogos. Hay, por supuesto, muchos otros que muestran pequeñas variaciones con respecto al grado de uniformidad, pero incluso cuando Jáuregui expresa la unidad tassiana con alguna libertad, se cuida de seguir su modelo colocando en posición prominente dentro del verso la forma afín de la palabra italiana principal:

è scritto: lungi, ah lungi, ite, profani far cupa e immedicabile ferita ch'a lui lasciò la fistola morendo d'invisibili fiamme: e questo dardo è però mal comune. Or non rammenti là dove essale un fumo pien di puzza che fa spesso cader di mano a Marte dar promettendo, a chi m'insegna a lui Nè la piaga di Silvia fia minore escrito está: lejos de aquí, profanos Oy he de hacer una profunda herida cuya zampoña le dejó, muriendo que toda espira llamas invisibles es daño al fin común; no se t'acuerda exhalan negro humo abominable derriba a Marte la sangrienta espada Ella me sigue y busca prometiendo será tan grande llaga la de Silvia

El porcentaje de versos españoles que conservan los acentos tónicos de Tasso en voces etimológicamente afines es muy alto, lo cual contribuye a mantener en la versión la fidelidad tonal de las palabras. Así, pues, en los oídos del lector español resonaba constantemente el eco del poema original, y se comprende que Cervantes y sus contemporáneos hayan tenido la ilusión de que la traducción de Jáuregui equivalía exactamente al texto italiano de Tasso: "donde felizmente pone en duda cuál es la traducción y cuál el original" (Don Quijote, II, 62). De manera análoga, y con mayor detalle, expresa Sedano sus alabanzas en el Parnaso español (t. 1, p. xxii):

Si los elogios fuesen sólo el objeto del presente juicio, ancho campo nos ofrecían para explayarse en ellos las ventaias y perfecciones que dan a esta Obra la preferencia sobre todas las Traducciones Castellanas. . Basta por último presentar esta pieza de examen para modelo de Traducciones y vergüenza de los que se arrojan a tradu- 
cir semejante especie de Poemas sin un talento y suficiencia semejantes, asegurando que si la Italia se gloría tan justamente con el original, sin envidia alguna debe gloriarse España con la copia.

Por su parte, Quintana declara en el Tesoro del Parnaso español (París, 1838, p. 291):

Nada hay que añadir a la opinión general, no sólo de España sino también de Europa, que reputa esta bella y elegante traducción como la más clásica de cuantas versiones poéticas se han hecho en castellano. Reimpresa en todas formas, leída, aprendida de memoria, recomendada y aplaudida a porfía, su crédito va creciendo en la proporción de que esta clase de trabajos se van haciendo más raros y los talentos que se ejercitan en ellos desaparecen. Aquí la lucha entre el original y el traductor era tan difícil cuanto que la obra tiene su mérito principal, tal vez el único, en las gracias del diálogo y del estilo y no en el interés de la acción ni en la disposición dramática de la fábula, uno corto y otra nula. Y con gloria suma de nuestra lengua y de nuestras letras se ve a Jáuregui en todos los trozos de esmero y de resalto estar a la par con su modelo y aun aventajarle a veces...

En su Biografia y estudio critico de Jáuregui (Madrid, 1899, pp. 899o), José Jordán de Urríes y Azara nos dice que "todos, absolutamente todos los escritores que hablan de [esta traducción]..., la ponderan en grado sumo", y cita en su abono las opiniones de Sedano, Quintana, Estala, Ticknor y J. L. Velázquez.

Mucha razón tiene Quintana cuando apunta la dificultad de reflejar fielmente las gracias del diálogo y del estilo de Tasso, no obstante que, al hacer esa afirmación, carece de detalles informativos. Los detalles se pueden encontrar en la mencionada carta dedicatoria de Jáuregui a don Fernando Enríquez. Consciente de los tropiezos que ha tenido que vencer, el poeta sevillano procura allí anticiparse a las censuras de los lectores haciendo una exposición de los principales obstáculos con que dice haber luchado.

En primer lugar -dice-, ha tenido que resolver el problema del coloquio pastoril, el cual "consiente muchas frases vulgares i modos de dezir umildes, i éstos en Italiano suelen ser tan diferentes de los nuestros, que parece cosa imposible transferirlos a nuestro idioma o propia locución". En segundo lugar -añade Jáuregui-, el toscano emplea algunas partículas que "en Castellano no ai manera que les corresponda". Y finalmente, aun descontando los obstáculos que ofrece el lenguaje pastoril, nota el traductor que el idioma italiano posee muchos vocablos tenidos por elegantes, pero de los cuales "nuestra poesía huye. . . por umildes". Pero a pesar de todo, dice en conclusión: "A sido trabajada esta pequeña obra con no poca diligencia, procurando ablandar sus asperezas de manera que no muestre 
la versión aver sacado de sus quicios el lenguaje castellano, i aunque muchas vezes se declaran concetos por diferentes palabras i modo..., no por esso pierden su gracia i gravedad, ni el verdadero sentido".

Es verdad que Tasso emplea no pocos "modos de dezir umildes" que difícilmente pueden traducirse con precisión al español; así, por ejemplo, algunos vocablos con terminaciones diminutivas (vermigliuzza, ritrosetta, pienotte, verginelli, tenerello, miserello), ciertos términos geminados (insieme insieme, spesso spesso, intiera intiera) y varios giros especiales (a mio grand'agio, ma che prò, buona pezza, prima ch'io vada in nulla, prendere a gabbo, e mi si chiuda lo spirto). Tales términos, sin embargo, forman parte del coloquio pastoril no porque éste sea un lenguaje especial dentro del italiano, sino porque los personajes que intervienen en el poema dramático son plebeyos y no cortesanos. Una de las partículas italianas más difíciles de traducir es pure, que aparece por lo menos veinte veces en el Aminta y que, en la mayoría de los casos, Jáuregui opta por no traducir. En algunos casos, el traductor parece haber tenido dificultades en hallar equivalencias exactas para giros como per mai sempre, quando mai, mentre. Es evidente, asimismo, que algunas expresiones que los italianos toleraban en composiciones elegantes podían sentirse como "umildes" al ser trasladadas al español, por ejemplo s'avviticchia, puzza, vesciche buge, spiedi, spunta, sforzata, stupro, crine, inaffiar (traducidas, respectivamente, por enlaza, abominable humo, viles trastos llenos, dardos, parece, ultraj[ada], ofensa, cabellos, bañar). Estos ejemplos, de ninguna manera exhaustivos, pueden servir como ilustración de las declaraciones de Jáuregui acerca de las dificultades que tuvo para hispanizar convenientemente el texto original.

En la dedicatoria, Jáuregui declara en forma bastante explícita su intención de elevar el tono "umilde" de Tasso y de reemplazar por otros aquellos vocablos italianos que, en su opinión, no producían una impresión de elegancia. En la práctica, sin embargo, Jáuregui suele llegar todavía más lejos. Reemplaza muchos latinismos del original por otros diferentes $-c u p a=$ profunda, fasto $=$ decoro, cupi$d o=$ lascivo, gentilezz $a=$ nobleza, antiveder $=$ pronóstico, leggiadria= gentileza, vermiglie $=$ purpureo, aura $=$ zéfiro-, y en algunos casos llega a latinizar innecesariamente las palabras italianas castizas empleadas por Tasso $-v$ uol saper $=$ inquirir, credo $=$ presumo, ritrosetta= esquiva, vi aggiungi=intervienen, trovato $=$ adquirido, desio=intención, nascosto $=$ oculto, crudele=rigoroso, d'accordo $=$ concertados, quel di fuore $=$ lo aparente, giovinetta $=$ ninfa, fatto=ejecutado, seguendo=persevera. A menudo duplica los términos sencillos de Tasso, adoptando así la costumbre de las parejas de sinónimos, tan fuerte en esa época. Algunos ejemplos: mi segue=me sigue $i$ busca, diporto $=$ solaz $i$ fiesta, felice $=$ feliz $i$ alegre, sciocca $=$ boba $i$ necia. . También, con bastante frecuencia, añade adjetivos a sustantivos que 
en el texto italiano aparecen sin ninguna modificación: donna= muger humana, tenebre = tinieblas espantosas, piaga=viva llaga, fascio=ceñidor infausto. Huelga decir que todo esto aleja a Jáuregui de la esencia del Aminta en lugar de aproximarlo a ella.

Quizá por ser algo demasiado evidente, Jáuregui no dice nada acerca de la profusión de palabras españolas agudas que constituían el mayor impedimento a su esfuerzo por darnos una exacta reproducción de los acentos tónicos de los versos del Aminta, particularmente los que están al final de los endecasílabos, donde no los toleraba la convención literaria de la época. Fue un tropiezo que a cada paso se le interpuso al sevillano desde el principio, y que mantuvo en tensión continua los notables recursos de su ingenio.

Si se considera el gran aprecio de que ha gozado el Aminta en su traducción castellana, extraña advertir que, con excepción de los brevísimos juicios de Sedano y de Ticknor, nadie, que yo sepa, ha intentado una comparación valorativa de la edición de 1607 con la de 1618 . Según Sedano, la revisión de 1618 fue tan rigurosa, que apenas se halla "verso sin reforma", de manera que puede considerarse como una versión distinta. Hay, en efecto, muchos cambios, pero no tantos como asegura Sedano, pues en realidad no llegan a más del diez por ciento del número total de los versos. A continuación añade el editor del Parnaso: "En este supuesto se presenta esta admirable Pieza [el texto que él publica] con toda la perfección, tomada de ambas ediciones, esto es, siguiendo la integridad de la primera y la corrección de la segunda" (op. cit., p. xxi). Así, pues, Sedano parece aceptar como mejoras todas las enmiendas llevadas a cabo por el traductor. Muy distinta es la opinión de Ticknor, el cual, en una nota escrita de su puño y letra en el ejemplar que poseía de la primera edición (conservado actualmente en la Boston Public Library), declara: "In the new edition -that of 1618-, of which I have two copies, the author made a great many changes, many of which are not improvements"'. Ticknor ve muchos defectos en las

2 Estas palabras, escritas entre 1862 y 1871 (año de la muerte del crítico), representan el último juicio de Ticknor. Las precede una cita del Diccionario de D. Hidalgo, s. v. Aminta: "Esta edición, Roma, 1607, que no he podido encontrar en ninguna de las bibliotecas de Madrid, es la primera". Con anterioridad, había dicho TICKNor en su History of Spanish literature, New York, 1849 , t. 2, p. 540: "The reputation of Jáuregui rests with the volume of poems he himself published in 1618. The translation of Tasso's Aminta with which it opens is elaborately corrected from the edition he had previously printed at Rome, without being always improved by the changes he introduced. But in each of its forms, it is probably the most carefully finished and beautiful translation in the Spanish language: marked by great ease and facility in its versifcation and especially by the charming lyrical tone that runs with such harmony and sweetness through the Italian". Con ligeros cambios, esta opinión pasó más tarde a la traducción española de Gayangos y Vedia, Madrid, ${ }_{1854}$, t. 2, pp. 
revisiones de 1618 , mientras que Sedano las consideraba casi sin tacha. ¿Es posible conciliar esta discrepancia crítica? Vamos a tratar de hacerlo en el análisis que sigue.

En la revisión léxica y estilística efectuada en la edición sevillana se rectifican varias traducciones erróneas o imperfectas. Por ejemplo, para el verso de Tasso: "Il Perso bea la Soma, il Gallo il Tigri", Jáuregui había dado originalmente esta traducción: "El Persa vea la Soma, el Franco el Tigris" (A), dejándose engañar, con toda seguridad, por la semejanza externa de bea y vea. Pero luego cae en la cuenta y corrige: "Beba la Soma el Persa, el Franco el Tigris" (B)3.

En cierto pasaje, Aminta alaba el timbre de la voz de Silvia y dice que sus palabras son mucho más dulces

che'l mormorar d'un lento fiumicello... o che'l garrir de l'aura in fra le frondi,

versos traducidos en A de la siguiente manera:

que el lento murmurar d'un arroyuelo... o qu'el bullir de zéfiro en las hojas,

donde el adjetivo lento, en vez de calificar al elemento visual, arroyuelo, viene a quedar junto a un elemento auditivo, murmurar, con una transposición que ciertamente no es desagradable; pero en el verso siguiente, bullir (elemento visual) se da como equivalente de garrir (elemento auditivo). En B, Jáuregui se da cuenta de esta incongruencia y en vez de bullir pone resonar, que, si no tan concreto como garrir, es un término más o menos cercano.

En "aquel qu'aquí por dios puede tenerse" (A) (Tasso: "colui che dio qui può stimarsi") faltaba la idea de estima o aprecio, y por eso se corrige: "el que en las selvas como a dios honramos" (B). Los tres conceptos del original tassiano "di gentile, / di mansueto e di cortese" se traducían imprecisamente "de hermosura, d'agrado i cortesía" (A), pero luego, con alguna mayor exactitud: "de agrado, de piedad y cortesía" (B).

He aquí otros casos en que el texto $\mathrm{B}$ es más fiel y preciso:

222-223: "La reputación de Jáuregui está fundada en el tomo de versos que imprimió en 1618. La traducción de la Aminta con que éste principia está corregida sobre la edición de Roma y presenta algunas variantes en las que no siempre anduvo el autor acertado: pero considerada en general, es la obra en su clase más bella y acabada de la lengua española, notable por la fluidez y soltura de la versificación y porque guarda exactamente la preciosa entonación lírica y la sin par dulzura de su original italiano".

${ }^{3} \mathrm{~A}=$ ed. de $1607 ; \mathrm{B}=$ ed. de 1618 . - Es curioso que Jáuregui no se haya dado cuenta de que era errónea también la traducción del verso "che la grandezza tua capir non ponno" (en el coro con que termina el primer acto); traduce en A: "donde caber no puede tu grandeza", y no hace ningún cambio en $\mathbf{B}$ 
A

orme

rozza scorza

case

lamentare

atti

pugna e pugnando

facondi detti

concorde voler planta

ásperas cortezas

morada

dolerme

costumbres

riñe $\mathrm{i}$ riñendo

delicado estilo

voluntad unánime
B.

huellas

rústicas cortezas

casería

quejarme

hechos

lucha y luchando

eloqüente estilo

voluntad conforme

En la arenga de Dafne a Silvia, con su exaltación ovidiana de la pasión amorosa en el mundo animal y vegetal, hay estos dos versos: "stimi dunque nemico / il monton de l'agnella", que rezan en A: "juzgas por enemigo / a dicha el carnerillo de la oveja"; el diminutivo carnerillo rayaba en lo ridículo, especialmente en contraste con oveja, pero el disparate queda remediado en B: "por ventura el carnero de la oveja". Sonaba igualmente incongruente, a propósito de los lobos que habían amenazado la vida de Silvia, la contaminación de 'beber' y 'comer': "tanto estavan al pasto embevecidos", según se parafraseaba en A el texto original: "tanto intenti erano al pasto"; en B, Jáuregui traduce literalmente: "tan atentos estaban a su pasto".

En otros casos, el amigo de Cervantes debió darse cuenta de que había empleado palabras que tenían dos o más acepciones, las cuales podrían chocar en la fantasía del lector, produciendo un efecto perjudicial desde el punto de vista estético. Por ejemplo, verdura puede significar 'frondas', pero, según anota Covarrubias, "comúnmente se toma por las legumbres que se crían en los huertos, como lechugas, rávanos, etc." A ello se debe seguramente que el verso de A, "dentro de su verdura" (Tasso: "dentro al lor verde") se haya modificado en $\mathbf{B}$, donde aparece "arboleda verde" en lugar de "verdura".

Tasso dice: “. . par ch'io sia certo / ch'ella se ne compiaccia o se ne doglia". En la versión A, "de cierto que se agrade o compadezca", el significado de 'inspirar lástima' que el verbo compadecer tiene en el contexto, está en conflicto con la otra acepción, 'compartir la desgracia ajena'. En B se rehace por completo el verso, y la idea se expresa con mucha mayor claridad: "que ha de causarle sentimiento o gozo".

El verso "E s'ella vuol che'l mio diletto sia" se traduce en A: “y si ella quiere que tu buena dicha", pero evidentemente la añadidura de buena a dicha no bastaba para borrar la acepción de 'suerte'. El remedio escogido en $\mathrm{B}$ consistió en reemplazar buena por alegre: " $\mathrm{y}$ si ella aguarda que tu dicha alegre", lo cual no parece un acierto.

En A se lee "al tiempo qu'entendió la dura nueva" (Tasso: "allora / ch'intese l'amarissima novella"); pero, disgustado seguramente por el exceso de nasales ("al tiempo qu'entendió..."), el traductor corrigió en B: "cuando escuchó la desdichada nueva”; sólo que aquí, al 
cambiar entender por escuchar, sacrificó la precisión en aras de la eufonía.

Convenir en el sentido de 'ser necesario' no es muy común, y puede tomarse fácilmente por 'ser a propósito', 'ser conveniente'. Así, el verso de A, "y también me conviene que lo cuente" (Tasso: "e pur convien narrarvelo"), se altera en B: "y me obligan también a que lo cuente", donde queda eliminada la ambigüedad.

En varios pasajes, la versión $B$ muestra el escrupuloso empeño de Jáuregui por dar mayor precisión lógica a las ideas de su modelo. Tasso había dicho de Silvia:

Poi si come ne gli occhi avesse un fonte, inaffiar cominciò co'l pianto suo il colui freddo viso.

El traductor prefirió no repetir la impropiedad que supone el reducir a una sola las fuentes de las lágrimas, e hizo este retoque: "Luego, como si fueran sendas fuentes / sus ojos..." Así también, en el prólogo del texto italiano dice el Amor que ha hecho una llaga en el pecho de Aminta "or son molt'anni"; Jáuregui tradujo primero fielmente, "ha muchos años" (A), pero luego corrigió "habrá algún tiempo" (B), considerando sin duda el hecho de que Aminta es todavía un zagal muy joven.

Los versos de Tasso: "che ciascun ne l'orecchio del vicino / mormorando diceva un suo secreto" aparecen así en A: "que cada uno por orden le decía / en la oreja un secreto a su vecino", donde la especificación por orden hace un poco más preciso el desarrollo del juego. En B, esa frase aclaratoria no debió de parecerle suficiente a Jáuregui, que añadió todavía un más casi pleonástico: “. . en la oreja un secreto al más vecino". De la misma manera, cuando en el texto italiano dice Aminta a Tirsi que le jure "di startene in disparte e non por mano / per impedirmi quel che son per fare", Tasso omite la acción intermedia de los pasos que tendría que dar Tirsi para llegarse a Aminta (aunque, naturalmente, está sobreentendida). En A, Jáuregui había traducido: "de estarte a un lado y no poner las manos", pero en B prefirió decir: "de estarte a un lado y no moverte un paso", donde lo que queda sobreentendido es el no poner las manos. Con la corrección ni se gana ni se pierde.

En una traducción que con tanta frecuencia utiliza formas etimológicamente afines a las del texto original, es sorprendente la relativa escasez de italianismos, sobre todo si se tiene en cuenta el ambiente italiano en que vivía Jáuregui en 1607 , y los gustos lingüísticos de la época. Hay dos que, con toda probabilidad, introdujo el traductor en el idioma castellano: trasoir y trasver, calcos del traudir y el traveder de Tasso. Otros italianismos que ya habían entrado en el léxico español en el siglo xvi y que reproducen en la versión 
A los términos del texto italiano, son avecinar, canora, presago, desdeñosa, al colmo, calar ('bajar'), bacante, formas que se conservan en $\mathrm{B}$, con excepción de desdeñosa (sustituida una vez por arrogante) y de calar (reemplazada por pender). Un préstamo más antiguo, vulto (A), traducción de volto, es cambiado luego en rostro (B). La forma desdeñosos (Tasso: dispettosi) aparece en A y se mantiene en B. En A, Jáuregui había traducido acertadamente argomenti por diligencias, pero en $\mathrm{B}$ decide adoptar la voz argumentos, que no parece tener en español la misma acepción que en italiano. Encontramos charlas y charlaria por ciance y ciancerebbe, y dos veces la palabra escuadra, una como traducción de schiera y otra en la versión parafrástica de "quell'alme in libertade avezze" (primer coro) ${ }^{4}$.

En un verso de A, "los pastores paisanos i estranjeros", encontramos ya la palabra paisanos, tomada directamenté del italiano (Tasso: "li pastori paesani e pellegrini"), con el sentido de 'convecinos', 'comarcanos', acepción bastante tardía. En B, Jáuregui prefiere traducir paesani por vecinos.

Es curioso encontrar tanto, en vez de tan, modificando a vezino. Se trata sin duda de un italianismo (como lo es también, posiblemente, el mismo adjetivo vezino en función de adverbio): "Ya que lo vi de mí tanto vezino" (A) (Tasso: "Or quando il vidi tanto/ vicin"). En B se corrige: "Ya que lo vi tan cerca de mí puesto".

Jáuregui sabía sin duda que el adjetivo vero ('verdadero') era voz castellana de vieja cepa, y así tradujo el verso "sani le piaghe mie con pietà vera": "sane este corazón con piedad vera" (A); pero, dándose cuenta quizá de que en su contexto podría tomarse fácilmente como italianismo, rehace el verso en $\mathrm{B}$ : "con fiel piedad mi corazón repare". Otro caso análogo es el cambio de tornarán (A) en volverán (B), al comienzo del primer acto. Así, también, temeroso tal vez de que se tomara el verbo albergar por italianismo, lo cambia en alojarse, sin darse cuenta, por lo visto, de que alojarse es voz más auténticamente italiana que albergar ${ }^{5}$.

Las pocas sustituciones de voces italianas que hallamos en $\mathrm{B}$ sólo nos permiten concluir que Jáuregui reaccionó de manera muy mo-

4 J. Terlingen, Los italianismos en español, desde la formación del idioma hasta principios del siglo xvii, Amsterdam, 1943, incluye escuadra, desdeño, desdeñoso, charla, charlaria y vulto entre los italianismos castellanos. No tengo seguridad absoluta en cuanto a los otros términos, que, como ya se ha dicho, representan neologismos introducidos en el siglo xvi; creo, sin embargo, que hay que considerarlos como italianismos (en el caso de calar, sólo en su acepción de 'bajar', 'descender'). Corominas apunta que présago aparece en la Galatea de Cervantes; para el uso de avecinar cita a Cetina, y para el de canora, a Lope y a Tirso; sobre bacante, dice en una nota: "En francés desde 1599"; pero, como se ve, aparece años antes en italiano, en el Aminta de Tasso.

5 Cf. también alloggiamento $=$ alojamiento, y véase TerLingen, op. cit., p. 174 . 
derada contra esa boga extranjera. En cambio, si consideraramos los italianismos que dejó intactos y otros que añadió en $\mathrm{B}$, concluiremos que el poeta estaba dispuesto a aceptar incondicionalmente en el léxico castellano la gran mayoría de las innovaciones lingüísticas italianizantes llevadas a cabo por los principales autores españoles del siglo xvı, desde Garcilaso hasta Cervantes.

Como ya hemos visto, se encuentran en $\mathrm{A}$ muchos ejemplos de voces sencillas traducidas mediante una pareja de sinónimos (diporto $=$ solaz $i$ fiesta, etc.), o de sustantivos escuetos que el traductor adorna con adjetivos (piaga=viva llaga, etc.). Todo esto tiene el efecto de añadir una intensificación expresiva a las palabras o conceptos. (En B no hallamos nuevos ejemplos de estos rellenos, quizá porque el traductor había agotado ya las posibilidades). También son frecuentes las traducciones cuyo significado literal se vigoriza para que tenga más dinamismo: así, en $\mathrm{A}$, la traducción de odio, odiare, odiato, odioso por aborrecimiento, aborrecer, aborrecido, abominable, de s'interni por encarne, o de fresche rose por rosas encarnadas. Hay ejemplos de esto mismo entre las expresiones retocadas de $\mathrm{B}$, pero el procedimiento se emplea aquí con mucha circunspección: come mi piace $=$ como me plaze $(\mathrm{A})>$ según mi antojo (B); appressare =arrimar (A) > juntar (B); si cerca=se busca (A) $>$ se pretende (B); "E'l ferro saria giunto a dentro" ="y llegara más adentro el hierro" (A) > "y pasara más adentro la punta" (B); dovuta $\cos a=\cos a$ debida $(\mathrm{A})>$ debida prenda $(\mathrm{B})$; odioso albergo $=$ odioso albergue $(\mathrm{A})>$ abominable albergue $(\mathrm{B})$. Más notable, porque modifica una imagen fundamental del texto italiano, es la transformación de sediento ciervo (A) en ciervo caluroso (B) (Tasso: assetato cervo). A Neptuno, el dios que sostiene la tierra y tiene el poder de sacudirla, lo había llamado Tasso scotitor de la terra; no se comprende por qué Jáuregui, después de traducir acertadamente que la tierra sacude (A), prefirió luego sustituir sacude por combate (B).

Es evidente en $\mathrm{B}$ el esfuerzo por lograr una mayor fluidez y tersura del verso. Jáuregui se empeña en limar las asperezas y en corregir las disonancias. Algunos ejemplos:

A

el lebrel de la liebre, amará el oso lo que Elpino contaba estotro día qu'en el infierno ai una cueva oscura sus pronósticos tristes infelices las ondas no alterava estotro día a domar un novillo, un oso, un tigre tomava ora una rosa ora un jazmín
B

el galgo de la libre, amará el oso lo que Elpino contaba el otro día que hay una oscura cueva en el infierno los pronósticos suyos infelices sus claras ondas serenaba un día a domar un novillo, un tigre, un oso de allí sacaba la azucena y rosa ${ }^{6}$

B Al hacer este cambio, Jáuregui se acordó seguramente del famoso verso de Garcilaso: "En tanto que de rosa y azucena..." 
se quede sin la infamia merecida i amor d'estotro sino a su carrera no poseyendo otras ningunas armas de hermosos ojos i de bellas manos i del amor agora goza el premio se quede sin la infamia que merece y amor del joven, sino a su carrera y no siendo señora de otras armas de bellos ojos y de lindas manos y agora logra del amor el premio.7

En varios lugares, los retoques llevados a cabo en $\mathbf{B}$ tienen por objeto acercar más la traducción al original, mediante el empleo de voces etimológicamente afines:

A

errar
impieghi
bagni
candido
torrente
gloria immortal
mostrò
gentilezza

andar
use
moje
blanco
corriente
perpetua gloria
enseñó
nobleza

andar

moje

blanco

corriente

enseñó
B

andar errando
emplee
bañe
cándido
torrente
gloria inmortal
mostró
gentileza

Para indicar 'un gran número', no hace falta decir que lo mismo se puede emplear mil que dos mil, expresión esta última muy frecuente en textos castellanos ${ }^{8}$. Así, cuando Tirsi le dice a Dafne que es "atta a tener mille fanciulle a scuola", Jáuregui tradujo el mille de Tasso por el castizo dos mil (A), pero luego prefirió ajustarse más escrupulosamente al original: "a dar a mil discípulas escuela" (B).

En varios pasajes, es visible en el texto $B$ el deseo de atenuar la sensualidad de ciertas expresiones del original y del texto A. Los versos "Dar promettendo a chi m'insegna a lei / o dolci baci, o cosa altra più cara" se traducen primero: “. . prometiendo / a quien me manifieste dulces besos"; pero en $\mathrm{B}$, los besos se convierten en un abrazo: ". . prometiendo / a quien me manifieste un dulce abrazo". En otro lugar, $i$ baci miei $=$ los besos mios $(\mathrm{A})>$ los halagos mios (B). Al mismo deseo de atenuación de lo sensual pueden deberse estos

7 A pesar de haber sido tan meticulosa la revisión de 1618 , todavía dejó Jáuregui sin ninguna reforma muchos versos criticables, como los dos que censura el "Liz. D. Luis de la Carrera”, autor del Anti-Jáuregui: “ „Esme forzoso andar huyendo de ella»: ¡Mire qué esme, i qué huyendo della! Pues éste lo enmienda: "Por ser puestas en uso ubas i trigos" (ed. M. Artigas, "Un opúsculo inédito de Lope de Vega", $B R A E, 12,1925, \mathrm{p} .602)$. Se podrían añadir otros casos análogos de versos poco eufónicos: "En este puesto, en éste, haré mi golpe”; "que las plantas, huyendo ser amante?”; “¿Qué estás diciendo? esfuérzate y conforta"; "te ve a turbar el sueño al preeminente"; "buscas derechamente disgustarla"; "persuasión importuna, pues mil veces"; "y anteponiendo mi servir continuo/a su retiramiento..."

8 Algunos ejemplos: gracias dos mil (Celestina, acto V), dos mil palos (ibid., $\mathrm{XIII),} \mathrm{dos} \mathrm{mil} \mathrm{vidas} \mathrm{(Lope} \mathrm{de} \mathrm{Vega,} \mathrm{El} \mathrm{perro} \mathrm{del} \mathrm{hortelano,} \mathrm{I,} \mathrm{xix,} \mathrm{y} \mathrm{La} \mathrm{dama}$ boba, II, XVI), dos mil azotes (Fuenteovejuna, acto II). 
cambios: sforzata =fuerçan $(\mathrm{A})>$ ultrajan $(\mathrm{B})$, y stupro=fuerça $(\mathrm{A})$ $>$ ofensa (B). Sin embargo, ya en A encontramos muchos ejemplos de esta misma austeridad o timidez del traductor: ardenti baci se convierte en vivo deseo, membre ignude en miembros bellos, seno en brazos, sforzerò (o rapirò) en quiero robar, rapisca en tome lo que pudiere. Así, pues, los nuevos cambios llevados a cabo en $\mathrm{B}$ no son sino el último retoque de una "expurgación" ya iniciada en A. Estas alteraciones pueden haberse debido al pudor de Jáuregui, o bien al deseo de satisfacer las exigencias de los censores inquisitoriales; en todo caso, reflejan muy bien el ambiente moralizante de la Contrarreforma.

Otro reflejo de la época, pequeño pero interesante, aparece en la alusión a la honra que se lee en uno de los coros, dentro de una evocación de la edad de oro: "quel che da'l volgo insano / onor poscia fu detto"; en A, Jáuregui decía: "éste qu’el enemigo vulgo insano / llamó después onor"; pero en B, teniendo quizá en cuenta el prestigio del tema del pundonor entre los nobles, prefirió decir: "a quien la urbanidad ${ }^{9}$ y el vulgo insano / llamó después honor".

"Forse ch'ei non è bello?", le pregunta Dafne a Silvia, refiriéndose al zagal Aminta. En A, Jáuregui tradujo literalmente: "¿No es, acaso, bello?"; pero la etiqueta social de la época se escandalizaba de que a un hombre se le llamara "bello" o "hermoso", y a ello se debe seguramente el retoque hecho en B: "¿No es un gentil mancebo?"'10

La comparación de las dos ediciones del Aminta castellano que aquí hemos hecho parcialmente nos permite llegar a la conclusión de que Sedano tiene más razón que Ticknor en cuanto a la superioridad del texto de 1618 . En efecto, con muy contadas excepciones -y no "muchas", como aseveró el crítico norteamericano-, los cambios que en él aparecen son verdaderas reformas que intensifican el valor artístico de la traducción; pero hay que añadir que esas correcciones no alteran en lo sustancial el carácter distintivo de la primera versión, o sea el cuidado con que se reproduce en español la musicalidad del poema italiano. Jáuregui demuestra que tenía un oído muy agudo y una sensibilidad singular para asimilar las delicadas cadencias de Tasso y para hacerlas reverberar copiosamente en cas-

9 Urbanidad como colectivo ('conjunto de cortesanos') opuesto a plebe o vulgo, parece ser neologismo exclusivo de Jáuregui.

${ }_{10}$ Garcilaso (Elegia I, v. 116) habla de la "gracia y hermosura" de don Bernardino de Toledo, lo cual le parece censurable a Herrera: "Más propio es esto... para alabar una dama que a un caballero" (palabras reproducidas en nota por T. Navarro, en su ed. de Garcilaso). En cambio Boscán, en su traducción del Cortesano, rehuye llamar hermoso o bello al varón: cf. MARgherita Morreale, Castiglione y Boscán: el ideal cortesano en el Renacimiento español, Madrid, 1959, t. 1, p. 239. 
tellano. Sólo cuando cotejamos los dos textos verso por verso podemos darnos cuenta de que la versión española no siempre se mantiene al mismo nivel que el original, y entonces nos parece que estamos leyendo un Aminta trasladado a un tono menor y con disonancias que aquí y allá rompen la eufonía de los versos. Pero si Jáuregui no nos dio una traducción perfecta en todos sus detalles -una traducción que, según Cervantes y otros, no podía distinguirse del original-, sí es evidente que sondeó, hasta los últimos límites que le ofrecía el genio de la lengua española, la posibilidad de ofrecernos una imagen fiel del poema italiano, que nos transmitiera, juntamente con su contenido, las vibraciones mismas del alma musical de Torquato Tasso.

JosepH G. FuCILLA

Northwestern University. 